\title{
Modelo sistémico de mejora continua para la optimización de procesos comerciales en empresas de telecomunicaciones: caso Grupo Visión Perú
}

\author{
Ricardo Jhunior Illescas Meza ${ }^{1 \mathrm{a}}$ \\ Universidad Peruana Unión, Perú1
}

Recibido: 21 de junio de 2016

Aceptado: 01 de diciembre de 2016

\begin{abstract}
Resumen
Esta investigación tecnológica enfoca la aplicación de la Metodología de los Sistemas Blandos conjuntamente con el ciclo de mejora continua, concretamente se ocupó en la descripción holística de los procesos comerciales existentes en Visión Perú, representando el "qué" y el "cómo" se realizan los procesos en el día a día, basados en componentes sociales, comerciales, de atención y de tiempo. Al culmino de las 7 fases se comparó los resultados obtenidos contra reportes anteriores para determinar que el Modelo Sistémico de Mejora Continua es eficiente en la optimización de procesos comerciales en empresas de telecomunicaciones: Caso Visión Perú.
\end{abstract}

Palabras clave: Procesos, Mejora Continua, Metodología de Sistemas Blandos.

\section{Systemic Model of Continuous Improvement for the optimization of commercial processes in telecommunications companies: the case of Grupo Visión Peru.}

\begin{abstract}
The current scenario for Peruvian telecommunications companies is changing in the commercial sense, according to the Supervisory Organization of Private Investment in Telecommunications (OPSITEL), it indicates that there is a greater development of the telecommunications market, the expectation of users is increasing and they have better services. Therefore, companies in the field have been seeking to improve or optimize their channels with the customer. Grupo Visión Peru is not new to this; they are positioned strongly in the Eastern Cone of Lima and have more than 18 years of experience in the telecommunications sector. Now, although Grupo Visión Peru is interested in optimizing its commercial channels, its management lacks the control of commercial processes, which is why in this technological research we focus especially on the application of the Soft Systems Methodology together with the Cycle of Continuous Improvement, concretely focused on the holistic description of the existing business processes in Visión Perú, representing the "what" and the "how" the processes are carried out on a day-to-day basis, based on social, commercial, service and social components as well as on time. At the end of the 7 phases, the results obtained from previous reports
\end{abstract}


were compared to determine that the Systemic Model of Continuous Improvement is efficient in the optimization of commercial processes in telecommunications companies: Caso Visión Perú.

Keywords: Processes, Continuous Improvement, Soft Systems Methodology.

\section{Introducción}

Las empresas de telecomunicaciones deben de estar en constante cambio para ser competitivos. En el caso del Grupo Visión Perú la carencia de un modelo de control holístico en los procesos comerciales, los limita a no ajustarse a constantes cambios en los que intervienen componentes sociales, de atención y de tiempo. Acuña (2013) aplicó el modelo sistémico de mejora continua para la optimización de procesos académicos, los objetivos alcanzados se centraron en el estudio de las características funcionales, en los aspectos estratégicos, de estructura, de procesos intermedios y de resultado de los procesos académicos de matrícula, de evaluación docente, evaluación discente y de graduación. Bijit (2008) y Valdez (2011) utilizaron la metodología de sistemas blandos para construir un modelo conceptual en el cual se apreció la integración y la interacción de todos los actores del entorno del sistema de gestión de residuos sólidos, y se brindó posibles soluciones a la problemática, basado en la metodología de sistemas blandos.

La realización de la investigación se centró en impactar eficientemente la optimización de los procesos comerciales de venta masiva, venta corporativa, cobranza y atención al cliente siendo estos vitales para el crecimiento del Grupo Visión Perú. Esto permitió tener una gestión de procesos más eficiente y obtener el máximo de funcionamiento al menor costo y menor tiempo, de tal manera que puedan ser competitivos en el mercado actual de telecomunicaciones en el Perú.

El objetivo principal de la investigación es determinar que el modelo sistémico de mejora continua es eficiente en la optimización de los procesos comerciales en empresas de telecomunicaciones: caso Grupo Visión Perú.

\section{Método}

Este estudio se enmarca dentro de una investigación científica en el campo administrativo tecnológico, pues se usa la conjunción de la Metodología de Sistemas Blandos de Checkland (1993) con el modelo de mejora continua de procesos de Deming (1989), concretamente se ocupa en la descripción holística de los procesos comerciales existentes en el Grupo Visión Perú, representando el "qué" y el "cómo" se realizan los procesos en el día a día, basados en componentes sociales, comerciales, de atención y de tiempo.

La investigación presenta un diseño no experimental, basada en el estudio de procesos, situaciones y cosmovisión y que está presente en la realidad del caso. Para tal efecto, se implementó un Modelo Sistémico de Mejora Continua para optimizar los procesos comerciales. Se aplicó una adecuación del diseño de preprueba y post-prueba. Se llevó un seguimiento del plan de mejoras aplicas en la realidad, validado por la estadística global de crecimiento y el plan estratégico del caso Grupo Visión Perú. 


\section{Descripción del Modelo Sistémico de Mejora Continua}

La investigación se llevó a cabo bajo el marco de las siete etapas de la Metodología de Sistemas Blandos (MSB) que redefine los procesos comerciales a partir de las situaciones problemáticas encontradas en el funcionamiento de los procesos comerciales, para ello se implementa el Modelo Sistémico de Mejora continua compuesto de las siguientes etapas:

\section{Etapa I: Situación no estructurada - planificar}

Es el punto de inicio donde se hace una intervención sistémica, se observa la situación problemática actual de cada proceso comercial analizando sus elementos y límites, mediante encuestas hasta técnicas analíticas.

\section{Etapa II: Situación estructurada - planificar}

Es aquí donde se define y grafica el sistema de referencia en esta etapa concatenaremos a todos aquellos que se encuentran involucrados con la problemática es decir aquellos que forman parte del sistema contenedor del problema y el sistema solucionador del problema. También se realiza un cuadro pictográfico, bajo los términos ya mencionados.

\section{Etapa III: Definiciones básicas - planificar}

En esta etapa se realizará una definición básica de los sistemas de actividad humana que forman parte del problema, en lo que una persona o grupo hace de la realidad. Cuando hablamos de definiciones hablamos de sistemas relevantes, cada sistema relevante constituye una definición básica.

\section{Etapa IV: Modelos conceptuales - planificar}

Cada definición básica genera un modelo conceptual, que no es sino la expresión, el lenguaje sistémico agrupación de verbos calificados y unidos gráficamente que nos indica la manera cómo se podría llevar a cabo el proceso de transformar la realidad social.

\section{Etapa V: Comparación de II versus IV - hacer}

Puesto que los modelos conceptuales son consecuencia de las definiciones básicas y elaboraciones mentales de procesos de transformación que pueden existir o no en la realidad, se requiere de un proceso o no de contrastación entre los modelos mentales y la realidad social que describen.

\section{Etapa VI: Cambios factibles y deseables - verificar}

En esta etapa detectaremos que cambios son posibles de llevar a cabo en los procesos comerciales del Grupo Visión Perú para ver si se podrá implantar en la realidad; estos cambios deben ser culturalmente factibles y sistémicamente deseables. 
Etapa VII: Implantación de cambios - actuar

Etapa donde se ejecuta de los cambios validados en la etapa anterior.

\section{Resultados y Discusión}

\section{Discusión de resultados en el Proceso de Venta Masiva}

El Modelo Sistémico de Mejora Continua es eficiente en la optimización del proceso de Venta Masiva en empresas de telecomunicaciones: caso Grupo Visión Perú, porque a partir de los cambios establecidos en los procedimientos intermedios de este proceso comercial, no se rebotaron más contratos, para el cierre del 2015 el Departamento de Ventas Masivas logro la meta establecida del 12\% y recuperó su indicador de tasa de crecimiento de años anteriores. Para el 2016 sin haber cerrado el año el indicador de del proceso ya va por los $13.21 \%$ y se espera que superarán la meta establecida para el cierre del 2016.

\begin{tabular}{|c|c|c|c|c|c|c|c|c|c|c|c|c|c|}
\hline \multicolumn{2}{|c|}{ INDICADOR } & \multicolumn{12}{|c|}{ TASADE CRECIMIENTO } \\
\hline \multirow{2}{*}{$\begin{array}{l}\text { FORMULA } \\
\text { DE } \\
\text { INDICADOR }\end{array}$} & \multirow{2}{*}{ FACTORES } & \multirow[b]{2}{*}{2014} & \multirow[b]{2}{*}{2015} & \multicolumn{10}{|c|}{2016} \\
\hline & & & & ENE. & FEB. & MAR. & ABR. & MAY. & JUN. & JUL. & AGO. & AVANCE & \multirow{4}{*}{$\begin{array}{c}\text { Pronostico } \\
\text { de Cierre } \\
\text { faborable } \\
\text { para el } \\
2016\end{array}$} \\
\hline \multirow{3}{*}{$\begin{array}{l}\text { Tasa de } \\
\text { Crecimiento } \\
\text { de abonados } \\
=\left(\left(\mathrm{N}^{\circ} \text { de }\right.\right. \\
\text { abonados } \\
\text { del periodo } \\
\text { actual } / \mathrm{N}^{\circ} \text { de } \\
\text { abonados } \\
\text { del periodo } \\
\text { anterior })-1)\end{array}$} & $\begin{array}{c}\text { Incremento } \\
\mathrm{N}^{\circ} \text { de } \\
\text { Abonados } \\
\text { (nuevos + } \\
\text { bajas) }\end{array}$ & 3942 & 5181 & 894 & 520 & 316 & 647 & 851 & 904 & 1341 & 909 & 6382 & \\
\hline & $\begin{array}{c}N^{\circ} \text { de } \\
\text { Abonados } \\
\text { del Periodo }\end{array}$ & 43127 & 48308 & 49202 & 49722 & 50038 & 50685 & 51536 & 52440 & 53781 & 54690 & 54690 & \\
\hline & Tasa \% & $10.06 \%$ & $12.01 \%$ & $1.85 \%$ & $1.06 \%$ & $0.64 \%$ & $1.29 \%$ & $1.68 \%$ & $1.75 \%$ & $2.56 \%$ & $1.69 \%$ & $13.21 \%$ & \\
\hline
\end{tabular}

Figura 1. Resultados en el Indicador del Proceso de Venta Masiva

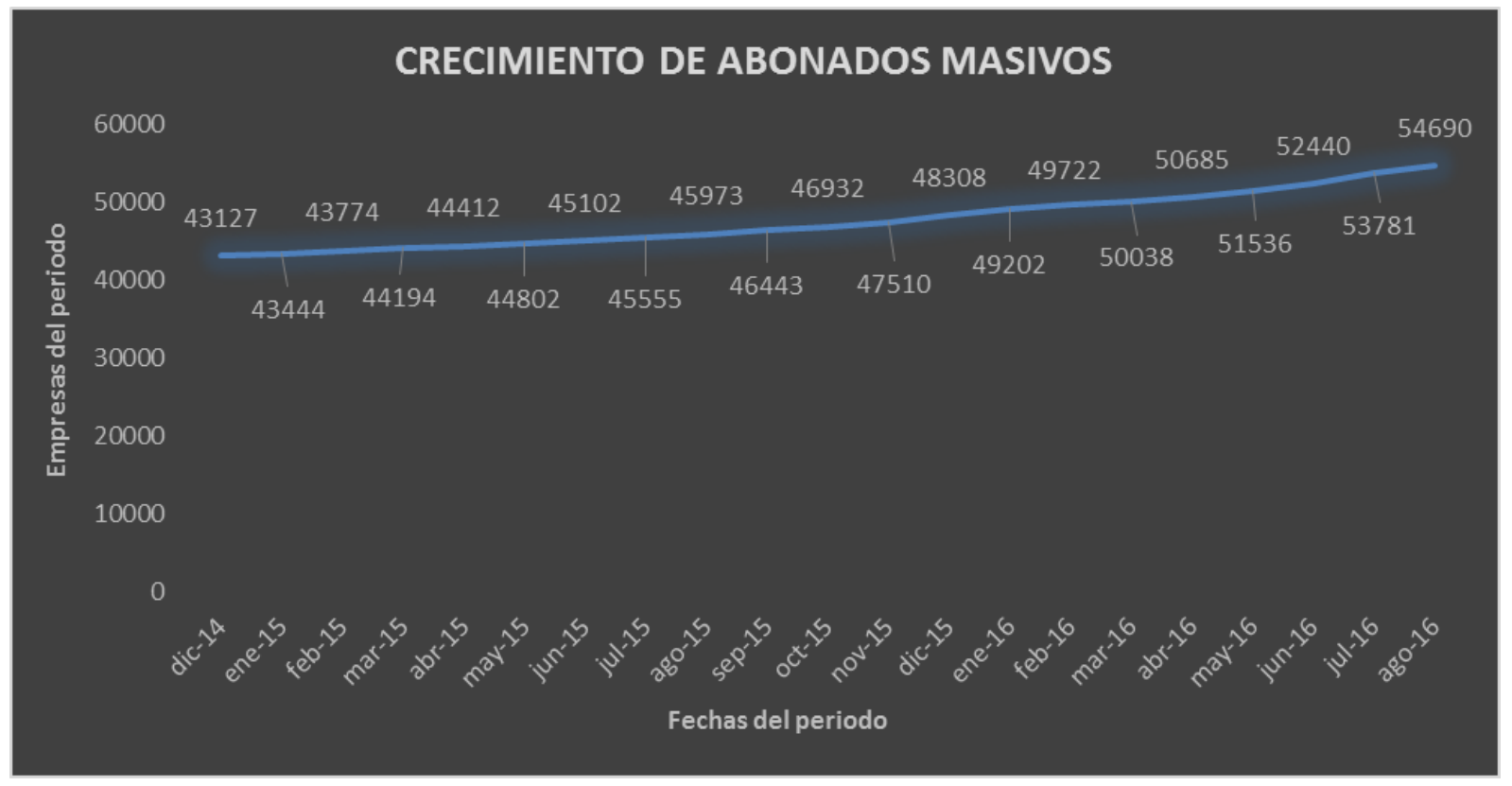

Figura 2 Resultados del crecimiento de abonados masivos.

Discusión de resultados en el Proceso de Venta Corporativa 
El Modelo Sistémico de Mejora Continua es eficiente en la optimización del proceso de Venta Corporativa en empresas de telecomunicaciones: caso Grupo Visión Perú, porque ayudo a determinar cómo hacer más con un actor menos en el proceso, permitiendo resolver la situación problemática y agilizar en tiempo el Proceso de Venta Corporativa del Grupo Visión Perú, que afectaba directamente a la posibilidad de clientes nuevos en el periodo. Las prospecciones pasaron de realizarse de 3 días a solo medio día. Según los resultados del cierre del año 2015 a partir de los cambios realizados se logró llegar a la meta de una Tasa de Adquisición del 15\% y superarla, aun teniendo 4 bajas corporativas en ese año se logró incorporar 12 empresas. En enero a pesar de ser el mes más bajo del año para el cierre de negocios corporativos, se logró gracias a la cantidad de prospecciones en rendir resultados en los meses siguientes, superando para el mes de agosto del 2016 las expectativas.

\begin{tabular}{|c|c|c|c|c|c|c|c|c|c|c|c|c|c|}
\hline \multicolumn{2}{|c|}{ INDICADOR } & \multicolumn{12}{|c|}{ TASA DE ADQUISICION } \\
\hline \multirow{2}{*}{$\begin{array}{c}\text { FORMULA } \\
\text { DE } \\
\text { INDICADOR }\end{array}$} & \multirow{2}{*}{ FACTORES } & \multirow[b]{2}{*}{2014} & \multirow[b]{2}{*}{2015} & \multicolumn{10}{|c|}{2016} \\
\hline & & & & ENE. & FEB. & MAR. & ABR. & MAY. & JUN. & JUL. & AGO. & AVANCE & \multirow{4}{*}{$\begin{array}{c}\text { Pronostico } \\
\text { de Cierre } \\
\text { faborable } \\
\text { para el } \\
2016\end{array}$} \\
\hline \multirow{3}{*}{$\begin{array}{c}\text { Tasa de } \\
\text { Adquisición= } \\
\left(\mathrm{N}^{\circ} \text { de }\right. \\
\text { empresas } \\
\text { nuevas del } \\
\text { periodo } / \mathrm{N}^{\circ} \\
\text { de } \\
\text { empresas } \\
\text { del periodo) }\end{array}$} & $\begin{array}{c}\mathrm{N}^{\circ} \text { de } \\
\text { Empresas } \\
\text { nuevas del } \\
\text { periodo }\end{array}$ & 4 & 8 & -1 & 2 & 0 & 4 & 2 & 1 & 2 & 3 & 13 & \\
\hline & $\begin{array}{c}\mathrm{N}^{\circ} \text { de } \\
\text { Empresas } \\
\text { del Periodo }\end{array}$ & 40 & \begin{tabular}{|c|}
48 \\
\end{tabular} & 47 & 49 & 49 & 53 & 55 & 56 & 58 & 61 & 61 & \\
\hline & Tasa \% & $10.00 \%$ & $16.67 \%$ & $-2.13 \%$ & $4.08 \%$ & $0.00 \%$ & $7.55 \%$ & $3.64 \%$ & $1.79 \%$ & $3.45 \%$ & $4.92 \%$ & $21.31 \%$ & \\
\hline
\end{tabular}

Figura 3. Resultados en el Indicador del Proceso de Venta Corporativa.

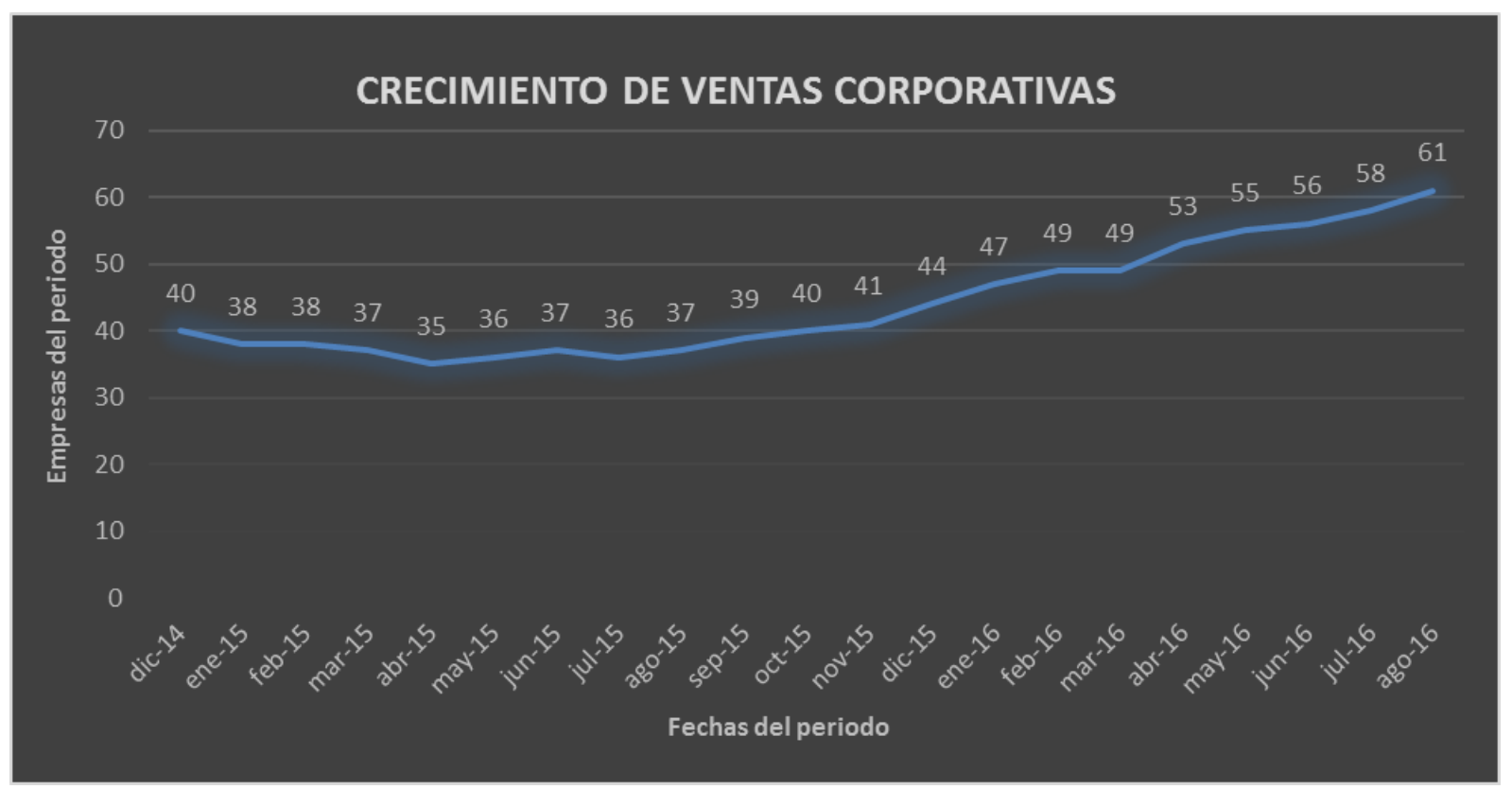

Figura 4. Resultados del crecimiento de clientes corporativos.

\section{Discusión de resultados en el Proceso de Cobranza}

El Modelo Sistémico de Mejora Continua es eficiente en la optimización del proceso de Cobranza en empresas de telecomunicaciones: caso Grupo Visión Perú, ya que se llegó a deducir eficientemente las medidas necesarias para aumentar la recaudación, todo esto pese a que la eficacia en acciones de cobranza se fueron reduciendo año a año y pese a que las acciones de mitigación no resolvían el problema, el modelo 
permitió encontrar cual era la mejor solución al menor tiempo, resultado fue un cambio radical en toda su estructura creando un nuevo departamento, para el cierre del 2015 no se pudo salvar el indicador ya que es una sumatoria anual, sin embargo gracias a las buenas practicas realizadas a partir de cuándo se implantaron los cambios estructurales para el año 2016 se percibe que es eficiente en $86.40 \%$ del indicador eficacia en acciones de cobranza y se pronostica que cerrarán el año favorablemente.

\begin{tabular}{|c|c|c|c|c|}
\hline \multicolumn{2}{|c|}{ INDICADOR } & \multicolumn{3}{|c|}{ EFICACIA DE LAS ACCIONES DE COBRANZA } \\
\hline $\begin{array}{l}\text { FORMULA DE } \\
\text { INDICADOR }\end{array}$ & FACTORES & 2014 & 2015 & $\begin{array}{c}\text { HASTA AGOSTO } \\
\text { DEL } 2016\end{array}$ \\
\hline \multirow{3}{*}{$\begin{array}{l}\text { Eficacia de las } \\
\text { acciones de } \\
\text { cobranza = } \\
\text { (Valor } \\
\text { recaudado / } \\
\text { Valor a } \\
\text { recaudar) x100 }\end{array}$} & $\begin{array}{c}\text { Valor Recaudado } \\
\text { en un Año }\end{array}$ & S/. $24,308,034.05$ & S/. $31,460,400.00$ & S/. $34,506,000.00$ \\
\hline & $\begin{array}{c}\text { Valor a } \\
\text { Recaudar en un } \\
\text { Año }\end{array}$ & S/. $35,343,172.89$ & S/. $39,584,353.64$ & S/. $39,937,785.37$ \\
\hline & EAC \% & $68.78 \%$ & $79.48 \%$ & $86.40 \%$ \\
\hline
\end{tabular}

Figura 4. Resultados en el Indicador del Proceso de Cobranza.

\section{Discusión de resultados en el Proceso de Atención al Cliente.}

El Modelo Sistémico de Mejora Continua es eficiente en la optimización del proceso de Atención al Cliente en empresas de telecomunicaciones: caso Grupo Visión Perú, ya que gracias al modelo se implanto labores de capacitación eficiente del personal de ATC y encuestas para medir resultados. logrando en el indicador tasa de retención la meta establecida para el 2015, para el año 2016 un 99.96\%, todo pinta favorablemente. En el indicador índice de reclamos, se redujo eficientemente colocándolo en una situación favorable en caso de alguna auditoria, se logró concretar la meta el 2015 y para el 2016 se pronostica que se mantenga.

\begin{tabular}{|c|c|c|c|c|}
\hline \multicolumn{2}{|c|}{ INDICADOR } & \multicolumn{3}{|c|}{ INDICE DE RECLAMOS } \\
\hline $\begin{array}{l}\text { FORMULA DE } \\
\text { INDICADOR }\end{array}$ & FACTORES & 2014 & 2015 & $\begin{array}{c}\text { HASTA AGOSTO } \\
\text { DEL } 2016\end{array}$ \\
\hline Índice de & $\begin{array}{c}\mathrm{N}^{\circ} \text { de Reclamos } \\
\text { Anuales }\end{array}$ & 287 & 229 & 131 \\
\hline de & Total Abonados & 43127 & 48308 & 54690 \\
\hline $\begin{array}{l}\text { Reclamos/Total } \\
\text { Abonados)*100 }\end{array}$ & IR \% & $0.67 \%$ & $0.47 \%$ & $0.24 \%$ \\
\hline
\end{tabular}

Figura 5. Resultados en el Indicador del Proceso de Atención al Cliente.

\section{Conclusiones}

1) Se determina que el Modelo Sistémico de Mejora Continua es eficiente en la optimización del Proceso de Venta Masiva en empresas de telecomunicaciones: caso Grupo Visión Perú. Debido a que se logró el incrementó eficiente en el indicador crecimiento de abonados masivo por los medios idóneos, logrando 
solucionar situación problema y mejorar los procedimientos de validación de clientes aptos, que afectaba directamente al indicador.

2) Se determina que el Modelo Sistémico de Mejora Continua es eficiente en la optimización del Proceso de Venta Corporativa en empresas de telecomunicaciones: caso Grupo Visión Perú. Debido a que el Modelo Sistémico ayudo a determinar cómo hacer más con un actor menos, optimizando eficientemente proceso.

3) Se determina que el Modelo Sistémico de Mejora Continua es eficiente en la optimización del Proceso de Cobranza en empresas de telecomunicaciones: caso Grupo Visión Perú. Debido a que se logró el máximo de funcionamiento de los procedimientos cumpliendo las metas en el menor tiempo, optimizando eficientemente el proceso.

4) Se determina que el Modelo Sistémico de Mejora Continua es eficiente en la optimización del Proceso de Atención al Cliente en empresas de telecomunicaciones: caso Grupo Visión Perú. Debido a que logro el máximo de resultados en el menor tiempo ayudando a deducir las medidas necesarias para mejorar los indicadores, cambiando los enfoques estratégicos y optimizando eficientemente el proceso.

\section{Recomendaciones}

Para el Caso del Grupo visión Perú no se realizó una comprobación (Diagrama de forrester u otra valida) de los modelos en la Etapa IV del Modelo Sistémico debido a que se validaba gracias al Plan Estratégico establecido por Visión Perú, siendo cualquier otra comprobación no factible para la cosmovisión del Grupo. Es posible comparar los resultados obtenidos en esta investigación con alguna nueva investigación de procesos no estructurados a realizarse en algún futuro del Grupo Visión Perú u otra del rubro de telecomunicaciones. Es factible que el Modelo Sistémico se pueda alinear a otras herramientas de gestión para procesos estructurados ejemplo BPM.

\section{Referencias}

Acuña Salinas, E. I. (2013). Modelo sistémico de mejora continua para optimización de procesos académicos: caso Universidad Peruana Unión. Lima: Universidad Peruana Unión.

Aguirre García, B. n. (2006). Calidad en el Servicio de Recojo de Basura de la Ciudad de Tarapoto Aplicando la Metodología de los Sistemas Blandos. Tarapoto.

Álvarez, W., \& Brito, M. (2001). Estudio sistémico de los Cursos Especiales de Grado de Ingeniería Química de la Universidad de Oriente, Núcleo de Anzoátegui. Universidad de Oriente.

Ancona García, I. (2005). Metodología de Sistemas Suaves: Un Enfoque para la Administración de Red de Telecomm/Telégrafos. Montemorelos - México: Universidad de Montemorelos.

Azabache Matos, A. (2012). Aplicación de la metodología de Sistemas Blandos a una Institución Educativa. Lima-Perú: Universidad Tecnológica del Perú.

Bijit S., J. A. (2008). Modelo de predicción de morosidad de la cartera de empresas de ENTEL S.A., usando redes neuronales. Santiago de Chile: Universidad de Chile. Escuela de Postgrado, Economía y Negocios. 
Checkland, P. (1992). Pensamiento de sistemas. México: Editorial Limusa.

Checkland, P. (1993). La Metodología de los Sistemas Suaves en Acción (Vol. Primera edición). México: Editorial Limusa.

Deming, W. (1989). Calidad, productividad y competitividad: la salida de la crisis. Madrid: Ediciones Díaz de Santos.

León Gonzales, R. J. (2013). Diseño de un Modelo Sistemico para el Diagnostico, Evaluación y Control del Plan de Seguridad Ciudadana de Piura Sustentado por el Plan Nacional de Seguridad Ciudadana 20132018. Piura-Perú: Universidad César Vallejo.

Parillo Condori, L. G. (2008). "Estudio del Sistema de tratamiento de los Desechos Electrónicos en la Ciudad de Tarapoto con la Metodología de Sistemas blandos".

Ramón Chavez, J. L. (2004). Aplicación de la metodología de sistemas suaves de Checkland para el diseño de un programa de formación docente de matemáticas: Caso de estudio Colegio Agustín de Hipona. México D.F: Instituto Politécnico Nacional de México.

Rodrigues Ulloa, R. (1994). La sistémica, los sistemas blandos y los sistemas de información. Lima: Universidad del Pacifico.

Senge, P. (1998). La quinta disciplina en la práctica. Bueno Aires: Editorial Granica.

Von Bertalanffy, L. (1993). Teoría General de Sistemas, Práctica de Sistema (Vol. Primera Edición). Mexico D.F: Grupo Noriega Editores.

Walton, M. (1992). "El Método de Deming en la Práctica, 6 compañías de éxito que usan los principios de control total de calidad del mundialmente famoso W.E.Deming". Barcelona: Grupo Editorial Norma. 